\title{
Parcours de praticien et savoir historique : quelles histoires pour un médecin polygraphe?
}

\section{Philip Rieder}

\section{(2) OpenEdition}

\section{Journals}

Édition électronique

URL : http://journals.openedition.org/edl/865

DOI : $10.4000 /$ edl. 865

ISSN : 2296-5084

\section{Éditeur}

Université de Lausanne

\section{Édition imprimée}

Date de publication : 15 mai 2016

ISBN : 978-2-940331-48-2

ISSN : 0014-2026

\section{Référence électronique}

Philip Rieder, «Parcours de praticien et savoir historique : quelles histoires pour un médecin 


\section{PARCOURS DE PRATICIEN ET SAVOIR HISTORIQUE: QUELLES HISTOIRES POUR UN MÉDECIN POLYGRAPHE? ${ }^{1}$}

En partant de fonds d'archives laissés par un médecin actif à la fin de l'Ancien Régime, l'objectif de cet article est d'explorer les pistes de recherche et les apports heuristiques d'une analyse de type biographique de ces fonds. Des problématiques quant au statut de la médecine, à l'économie de la pratique, à la relation thérapeutique et au rapport entretenu par le praticien avec le savoir médical seront abordées. Au cours du travail, des questions méthodologiques émergent. Quelle est la spécificité des données issues de la confrontation des données individuelles avec un contexte spécifique? Est-il possible, à partir du cas singulier, de penser autrement l'histoire du groupe social dont il fait partie? Quelles autres réalités historiques sont accessibles à partir de l'histoire d'un sujet?

Louis Odier n'est pas de ceux dont la contribution à la science médicale moderne a été déclarée décisive par l'histoire traditionnelle et positiviste de la médecine. Il fut un médecin respecté à Genève où il œuvra entre 1774 et 1817 . Sa renommée médicale s'étendait dans le bassin lémanique et au-delà. Ses travaux de médecine étaient connus en France, en Grande-Bretagne et dans de nombreux centres médicaux européens. Sur le plan scientifique, on lui reconnaissait la paternité de la découverte de l'efficacité du magister de bismuth pour les maux d'estomac, de travaux sérieux sur l'histoire clinique de l'hydrocéphale, d'études sur

I. Je remercie Danièle Tosato-Rigo et Michel Figeac pour leurs commentaires constructifs sur des versions antérieures de cet article. La recherche nécessaire à l'élaboration de cet article a été rendue possible par un subside du Fonds national suisse de la recherche scientifique (FNS 100011-122584). 
l'espérance de vie et nombre d'autres sujets médicaux ${ }^{2}$. Louis Odier fut un médecin distingué, un praticien reconnu, mais non une célébrité. Ce n'est par conséquent ni l'originalité ni la normalité de son parcours qui font son intérêt historique. Il faut le chercher ailleurs, dans l'importance des fonds manuscrits où se trouvent précieusement conservés des ordonnances médicales, des journaux personnels, des autobiographies, des carnets de visites, des comptes rendus de séances, des résumés de livres, des récits de cas, des procès-verbaux, des expertises judiciaires, des pétitions, des factures, des comptes, des témoignages, des requêtes, des mémoires, des lettres. Son œuvre publiée n'est pas en reste: elle compte un livre de médecine, plusieurs traductions, des éditions de textes, des articles scientifiques, des chroniques journalistiques et même un abécédaire ${ }^{3}$.

Manuscrits et publications témoignent de l'importance de l'écriture pour leur auteur qui s'impose comme un homme de lettres et un raconteur de soi. Tous ces documents ne sont pas des egodocuments stricto sensu, mais tous sont l'occasion pour Odier de se raconter et de se donner à voir. Ils sont l'indice d'une ambition qui se confirme dans les déclarations du jeune adulte dans sa correspondance. A peine docteur, il rapportait à sa fiancée son "ambition de ne rien négliger pour acquérir de la gloire», précisant:

Auparavant, il est vrai que mon imagination s'élançait souvent sur les ailes de la gloire dans l'avenir le plus brillant, mais ce n'est que depuis l'heureuse époque de notre amitié et de notre union que votre petit docteur a travaillé pratiquement et avec activité à se rendre un jour grand homme $e^{4}$.

Sept ans plus tard, il exprime la même aspiration à sa seconde fiancée, l'assurant «ne rien négliger à l'avenir, pour mériter de plus en plus la confiance du public, l'estime des honnêtes gens, et la considération de tout le monde, et pour obtenir de la postérité, le titre de grand homme $[\ldots]{ }^{5}$. Les efforts qu'il déploiera pour être admis à la Société

2. Voir P. Prevost, Notice de la vie et des écrits de Louis Odier.

3. L. Odier, Les honoraires médicaux et autres mémoires d'éthique médicale, p. 173 sq.; L. Gautier, La médecine à Genève jusqu'à la fin du XVIII' siècle, p. 538-544.

4. Bibliothèque de Genève (désormais BGE), Ms fr 4151, Louis Odier à Susanne Baux, le 2 mars 1772. Voir aussi sa lettre à la même le 21 novembre 1772 .

5. BGE, Ms fr 5656, Souvenir sur le Dr Odier, t.3, p. 155. 
Royale de médecine ${ }^{6}$ et, plus tard, à l'Institut de France, confirment la pérennité de cet objectif 7 . Louis Odier veut laisser sa marque, rédige des textes autobiographiques, conserve ses mémoires et dissertations d'adolescent, archive ses copies de lettres, notes et brouillons. Il a mené une "vie biographique» (biographical life), selon l'expression de Thomas Söderquivist, en conservant des indices sur son parcours et en préparant la voie à ses biographes ${ }^{8}$. La pratique était courante parmi les médecins connus de la période qui précède directement l'ère de la médecine scientifique $^{9}$. Ce groupe se distingue avant tout par sa production publiée et par la propension de ses membres à s'écrire. La reprise peu critique de leurs textes, de fait l'indice d'une campagne de self promotion, par des biographes habités d'un projet téléologique, explique le discrédit qui plane sur la biographie aujourd'hui dans le champ de l'histoire de la médecine. Cette déconsidération est renforcée par des problèmes plus généraux inhérents à l'écriture biographique comme l'importance donnée à un récit habité par ce que Pierre Bourdieu a si bien défini comme "l'illusion biographique», soit la notion naïve mais répandue que la vie d'un individu serait cohérente et inscriptible dans un récit ${ }^{10}$. De fait, l'articulation d'un travail d'histoire autour d'un acteur historique, soit en adoptant une approche biographique, s'accompagne du risque de voir les problématiques développées minimisées au bénéfice du récit de vie lui-même. En clair, les livres portant sur des médecins historiques dans lesquels des postures professionnelles anciennes sont reconstruites et des problématiques historiques novatrices abordées souffrent du soupçon que la trame narrative adoptée puisse fausser la réalité historique relatée. L'apport historique de tels ouvrages s'en trouve négligé. Dans un commentaire à propos de son livre sur Laennec, Jacalyn Duffin dit avoir constaté, non sans surprise, que dans cette contribution à l'histoire de la

6. Académie Royale de médecine, SRM, boîte 185, dossier 15/1-3.

7. Bibliothèque de l'Institut de France, Ms 3190/2.

8. T. Söderquivist, Science as autobiography, p. XVIII. Voir aussi M. J. Nye, "Scientific Biography», p. 327.

9. Parmi d'autres: F. Loux, Pierre-Martin de la Martinière un médecin au XVIII siècle, N. G. Siraisi, The clock and the mirror; E. Le Roy Ladurie, Le siècle des Platter; J.-P. Goubert, Médecins d'hier, médecins d'aujourd'hui; W. Balster, Medizinische Wissenschaft und ärzliche Praxis.

Io. P. Bourdieu, «L'illusion biographique». 
clinique à travers une vie, les éditeurs ne voyaient qu'une biographie ${ }^{11}$. En prenant en compte l'ensemble de ces difficultés, et afin d'aller au-delà des querelles stériles sur la nature du récit biographique, l'objectif de cet article est d'explorer concrètement ce qu'un parcours de médecin bien documenté peut apporter à l'histoire de la médecine.

En abordant un tel questionnement, il convient de rappeler que les apports de l'historiographie récente sont loin d'être négligeables. Des cas de soignants particuliers se voient utilisés comme des exemples illustratifs, indiquent des cas extrêmes, signalent l'émergence d'une attitude, attirent l'attention sur la nature théorique de la médecine pratiquée ou, plus simplement, révèlent un niveau de réalité autrement inaccessible... ${ }^{12}$ Une première constante dans les titres de publications ne manque pas d'intérêt. Les noms des personnalités qui en constituent le centre tendent à être placés en sous-titre, au profit des problématiques abordées. Une seconde caractéristique réside dans l'accès, à partir du cas étudié, à une autre échelle historique. Le pieux praticien Philippe Hecquet, par exemple, donne à voir une posture extrême de médecin chrétien au début du XVIII ${ }^{\mathrm{e}}$ siècle, tout en offrant un aperçu de ce à quoi il s'opposait, soit à un corps médical intégré dans la haute société, vivant ostensiblement dans le luxe et le paraître ${ }^{13}$. Les avantages de l'approche sont nombreux. La concentration sur une problématique permet, d'abord, d'évacuer le problème de la cohérence narrative tissée autour d'une vie. Ensuite, l'échelle d'analyse choisie est un moyen de se confronter à une altérité, une posture proche de celle d'un anthropologue face à un des membres de la société qu'il étudie: elle oblige à remettre en question ses concepts et ses valeurs. Cela dit, le format de l'article, celui qui est le plus souvent adopté, incite à réduire le parcours étudié à une problématique particulière. Dans le cas des fonds Odier, un grand nombre de thématiques possibles émergent à la lecture des corpus. Certaines ont trait à la vie culturelle, d'autres à la vie émotive, d'autres encore à l'histoire de soi ou à celle de la médecine. La multiplicité des possibilités s'accompagne de difficultés pour l'historien qui se trouve

II. J. Duffin, «"La mauvaise herbe”", p. 191 sq.

I2. Voir parmi d'autres: H. Steinke, "Der Junge Arzt und seine Patienten»; R. Mandressi, «Le passe, l'enseignement, la science»; S. De Renzi, «Medical competence, anatomy and the polity in seventeenth-century Rome».

13. L. Brockliss, The Medico-religious Universe of an Early Eighteenth-Century Parisian Doctor. 
contraint de reconstituer une série de contextes, ne serait-ce que pour évaluer l'intérêt historique des contenus. Le parti pris ici est de regrouper plusieurs problématiques proches, liées à la persona professionnelle du médecin, soit sa vocation, sa vie économique et son rapport au savoir, afin de mettre à l'épreuve l'approche et de tracer l'esquisse de la biographie d'une pratique médicale.

\section{Choix professionnel ou vocation?}

La vocation médicale représente une composante importante de l'identité professionnelle à la fin de l'Ancien Régime ${ }^{14}$. Comment une vocation se déclare est alors une question importante en raison de la qualité héréditaire de l'activité: près de $50 \%$ de l'élite médicale choisie par Vicq d'Azir ${ }^{15}$ pour ses éloges émane du milieu médical. Les motivations de candidats médecins tel Louis Odier, dépourvus d’appuis médicaux, sont essentielles pour saisir celles du corps médical croissant à la veille de la Révolution, dont les nouveaux venus proviennent pour la plupart, comme lui, de sphères sociales étrangères à la profession ${ }^{16}$. De prime abord, la question est simple. A l'époque de ses études déjà, Odier affirmait à qui voulait l'entendre qu'en se formant à la médecine il n'avait fait qu'obéir à son père. L'injonction aurait été proférée un jour de la fin de l'année 1766. La scène est rapportée dans une lettre à son ami Stephen Pellet. Odier cite verbatim la question de son père: "Comment voulez-vous gagner votre vie?» Il raconte aussi la suite du dialogue: «Je lui répondis que je m’en remettais à lui pour cela, que je voyais trois voies à moy ouvertes, le commerce, le droit, et la médecine; que je le priais de choisir. " La décision survient alors spontanément: "Il ne faut penser ni au commerce, ni au droit, me dit mon père. "Eh bien! Je serai médecin! Répondis-je. " " 17

Tout semble être dit. Il n'y a pas de raison de douter que cette scène ait eu lieu. Il est possible, par contre, de remettre en question

I4. O. Guillemain, «Devenir médecin au XIX” siècle», p. 111-113.

I5. D. Roche, "Talents, raison et sacrifice», p. 870.

I6. Le nombre d'étudiants en médecine progresse à cette époque dans différents pays européens. Voir Ph. Rieder, M. Louis-Courvoisier, «Enlightened Physicians», p. 579 sq.

I7. BGE, Ms 5642/4, 13, Louis Odier à Stephen Pellet, [Edimbourg], 26.11.1768 
l'interprétation qu'en fait Odier à l'intention de son ami. Afin d'en saisir les enjeux, il faut remonter à l'époque de l'enfance d'Odier. Sa vocation était alors de devenir pasteur. Il déclamait des sermons, apprenait sérieusement ses devoirs de religion et ambitionnait de remporter chaque année le prestigieux prix de piété décerné dans l'école genevoise ${ }^{18}$. Les motivations du jeune homme n'apparaissent pas explicitement, mais elles ne sont guère difficiles à deviner quand on sait que son père était pieux, qu'il aurait voulu étudier la théologie et idolâtrait le pasteur du quartier... ${ }^{19}$ Dès lors, pourquoi renoncer à cette première vocation? Une lecture attentive des manuscrits contemporains de l'époque où il modifie son choix révèle que ce dernier ne s'effectua pas du jour au lendemain et qu'il marque certainement une prise de distance par rapport à son père. Un carnet, intitulé "compositions» et contenant des rédactions d'Odier adolescent, permet d'établir la chronologie des événements. En 1765, âgé de 17 ans, il rédige encore des sermons. Ses préoccupations le portent progressivement de sujets purement théologiques vers des thématiques sociales et morales. Il disserte sur le travail, le mariage et la lubricité. Son «Discours sur les objets du 7e commandement: Tu ne paillarderas pas» ${ }^{20}$ est nourri d'idées inspirées par ses lectures bibliques, mais également de notions médicales tirées d'un ouvrage du médecin lausannois Samuel Auguste Tissot dont le nom figure dans la marge du cahier ${ }^{21}$. C'est en soi une indication de la montée en puissance du discours hygiéniste face au discours religieux ${ }^{22}$. Est-ce alors, en prenant conscience de l'importance prise par des arguments médicaux dans sa dissertation qu'Odier se découvre un intérêt pour la médecine? Le lien n'est pas avéré, mais possible: la composition suivante, datée du 9 novembre 1765 est intitulée "Sur la médecine». L'adolescent y développe ses idées sur les qualités du médecin et sur l'utilité de la médecine. La lecture faite de la personne du médecin est pragmatique: le médecin aurait avant tout le caractère prudent et ferme, les sens développés, la figure agréable, la voix claire et distincte, l'amour du travail et de l'étude:

I8. BGE, Ms fr 4152, Louis Odier à Andrienne Lecointe, [Genève], s.d. et Ms fr 4153, Louis Odier à Andrienne Lecointe, [Genève], s.d.

I9. BGE, Ms fr 4153, Louis Odier à Andrienne Lecointe, [Genève], s.d.

20. BGE, Ms fr 5644 / Cahier rouge, Compositions Louis Odier 1765.

2I. Il s'agit de: S. A. Tissot, L'onanisme.

22. Voir S. Pilloud, Les mots du corps, p. 40-44. 
qu'il sache à fond l'anatomie, la chymie et la botanique, la physique et la mécanique, qu'il entende un peu l'astronomie, la géographie, l'histoire, qu'il sache le grec et le latin en fait de langues mortes, le français, l'allemand, l'anglais et l'italien en fait de langues vivantes, qu'il n'ignore pas la théologie, la morale, la logique, les mathématiques ${ }^{23}$.

Certaines de ces qualités sont attendues du médecin: les capacités scolaires, une propension au travail et le caractère prudent et ferme, par exemple. Font défaut, par contre, dans cette liste des qualités morales telles le dévouement pour autrui, la modestie et la volonté d'être utile à ses semblables, autant de traits revendiqués par la profession qui rapprochent le médecin de l'ecclésiastique. Une autre absence à relever est celle de la notion de piété, pourtant régulièrement invoquée par des générations antérieures de médecins ${ }^{24}$. La piété d'Odier ne laissant aucun doute, on est en droit de se demander si cette absence n'apporte pas une confirmation à la thèse de la séparation précoce dans le monde francophone - sous l'égide des Lumières - entre la sphère religieuse et celle de la santé. ${ }^{25}$

En 1765, l'exercice d'Odier est scolaire. Il ne doute pas à cette date de la prééminence de la théologie comme en témoigne un texte rédigé au début de l'année 1766, un "Dialogue entre Spanheim, Boërhaave et Patru» dans lequel il disserte sur la valeur respective des professions de juriste, de médecin et de théologien. Le théologien gagne la partie haut la main. Quelques mois plus tard pourtant, au mois de mai 1766, l'adolescent rédige une "Confession de foi » inspirée par la «Profession de foi du vicaire savoyard " insérée par Jean-Jacques Rousseau dans son Emile $(1762)^{26}$. L'équilibre particulier entre raison et foi qui y prévaut a sans doute plu à l'étudiant, formé au sein de l'Académie de Genève par des philosophes et des naturalistes acquis aux idéaux des Lumières. La réaction outrée du pasteur de quartier à qui Odier avait soumis son texte

23. BGE, Ms fr 5644 / Cahier rouge, Compositions Louis Odier 1765, p. 88. La liste est proche de celle de Bernard de Gordon (XVe siècle). L. Demaitre, "Theory and Practice in Medical Education at the University of Montpellier», p. 122.

24. La piété était une qualité traditionnelle du médecin, notamment dans des contextes germaniques. T. H. Broman, «Rethinking Professionalization», p. 832.

25. M. Stolberg, Homo patiens, p. 54.

26. Texte reproduit dans: BGE, Ms fr 5654, Amélie Odier, Souvenirs sur la vie privée de Louis Odier (t.1), p. 13-16. 
suffit à le dégoûter de l'idée de poursuivre dans cette voie. La carrière pastorale est ainsi écartée. Restent en été 1766 les trois possibilités énoncées dans l'entrevue avec son père rapportée par Odier. Le commerce, activité du père, aurait été la solution de facilité, si les trois autres fils Odier n'avaient pas déjà choisi cette voie, assurant la succession professionnelle paternelle. D'ailleurs si le jeune homme avait voulu choisir cette voie, il aurait eu intérêt à interrompre ses études plus tôt. Agé de dix-huit ans, Louis Odier a déjà à son actif un passé scolaire brillant et une mâ̂trise du latin et du grec qui ne lui auraient guère été utiles dans le commerce. La famille Odier et l'adolescent lui-même visent une formation académique. Restent donc le droit et la médecine. Le droit n'était de toute évidence pas le domaine de prédilection d'Odier qui l'avait placé en troisième position dans son «Dialogue" de $1766^{27}$. C'est d'ailleurs une profession qui requiert une certaine aisance orale qu'il ne pense pas posséder et qui lui serait même inaccessible en raison d'un défaut de prononciation qu'il se reconnait ${ }^{28}$. Par ailleurs, à Genève, le droit était un premier pas dans la carrière publique, une voie fermée à Odier en raison de l'établissement relativement récent de la famille dans la ville, Antoine, le père, étant lui-même un réfugié français.

Avant même l'entrevue rapportée par Louis Odier, la médecine était donc la seule option sérieuse: un défi académique que le jeune homme pouvait espérer relever, avec, par ailleurs, la possibilité de gagner sa vie et d'acquérir le prestige social auquel la famille aspirait. Un aspect frappant dans les textes d'Odier sur la médecine et sur sa propre vocation est - à l'instar de ce qu'il écrivait dans sa composition de 1765 - l'absence des qualités revendiquées par la corporation elle-même: le dévouement, l'altruisme, la compassion et l'humanité; des qualités qui sont pourtant bien présentes dans l'image publique du médecin telle qu'elle se dessine dans les éloges de médecins célèbres ${ }^{29}$. La raison de l'omission n'est pas claire. Ces qualités sont-elles trop évidentes? Le jeune homme

27. Voir plus haut p. 67.

28. Voir les lettres adressées à Andrienne Lecointe (23 avril 1779 et s.d.) insérées dans BGE, Ms fr 5655, Amélie Odier, Souvenirs sur la vie privée de Louis Odier (t. 2).

29. Ces qualités (la bonté, la patience et la compassion) sont énumérées par Gregory à cette même époque. J. Gregory, Lectures on the Duties and Qualifications of a Physician. Pour August Unzer, médecin à Hambourg, le médecin devait posséder un bon cœur, la capacité de raisonner et de l'érudition. T. H. Broman, «Rethinking Professionalization", p. 844. 
estime-t-il déjà les posséder? L'absence d'une autre variable est tout aussi intrigante: le prestige des études académiques n'est pas mentionné. Or, le jeune Odier cherchait de toute évidence une voie qui lui permettrait de se mettre en avant. L'ambition sociale serait-elle, elle aussi, une caractéristique évidente du futur médecin en devenir? A défaut de pouvoir répondre clairement à la question, le cheminement de l'adolescent dans sa quête professionnelle incite à se méfier des affirmations des acteurs quant à leurs propres motivations. De toute évidence, à un moment donné, Odier cherche à se donner à voir comme un fils obéissant. La réalité, j'espère l'avoir montré, est bien plus complexe. Dans le contexte culturel des Lumières et du discrédit qui affecte la religion, sa réorientation de la théologie vers la médecine n'a rien d'étonnant.

\section{Economie d'une pratique}

Dans la scène de 1766 rapportée plus haut, Antoine Odier demandait à son fils comment celui-ci entendait gagner sa vie. Depuis l'extérieur de la profession, la pratique médicale pouvait être vue comme une source possible de revenus conséquents: à partir du Moyen Age, nombre de médecins étaient connus pour gagner beaucoup d'argent ${ }^{30}$. Le corps médical lui-même se voyait constamment accuser de n'être intéressé que par la rémunération. Est-ce à dire que le médecin d'Ancien Régime pouvait gagner facilement sa vie? Les Odier eux-mêmes n'en sont pas sûrs. Antoine Odier raisonne en se fondant sur les apparences:

Tous nos amis médecins ont fait de bonnes maisons. Si on n'est pas payé comme en Angleterre, les dépenses y sont beaucoup moins fortes, et un médecin honnête homme, entendu, religieux et charitable y est toujours estimé et considéré ${ }^{31}$.

Peu d'éléments historiques soutiennent une telle impression, nombre de médecins provenant de milieux aisés, ce qui les rendait financièrement indépendants avant d'entrer dans la pratique. Dans ses lettres, tout au long de sa carrière, le praticien Odier se donne à voir comme un homme

30. Voir N. G. Siraisi, Medieval and early Renaissance medicine, p. 17; G. Jubert, "L'homme Renaudot", p. 20-22.

3I. BGE, Ms fr 4152, Antoine Odier à Louis Odier, Genève, le 17.10.1772. 
désargenté et se plaint de difficultés économiques. A la veille de s'établir, il estimait sa fortune personnelle à 33'000 livres tournois (20'000 louis courants de Genève) et envisageait une vie simple dans une masure rurale tout en s'inquiétant d'épouser une femme sans biens. ${ }^{32}$ Plus tard, il scrute la moindre variation de ses revenus médicaux et s'inquiète de ne pouvoir nourrir les siens. "Je n'ai reçu pendant tout le mois de juin que 182 louis courants 4 sols et 9 deniers", écrit-il à sa femme en été 1787 , «et je suis en arrière de plus de 300 louis courants de ce que j'avais l'année passée dans cette saison. Cela m'inquiète pour l'avenir [...]" ${ }^{33}$.

Quelle était la situation financière de Louis Odier? L'étude de ses comptes et des actes relatifs à sa fortune montre qu'il n'est pas, au moment de son établissement, fortuné au point de pouvoir se dispenser de travailler. Il bénéficie d'une somme qui, bien investie, lui permettait de s'acquitter du coût de son logement et d'une partie de sa subsistance. Quant aux revenus dérivés de la pratique, la compétition entre docteurs était rude et les malades payaient mal et irrégulièrement ${ }^{34}$. Odier s'en plaint dans les réflexions sur les honoraires médicaux qu'il partagera avec ses collègues à la fin de sa carrière (1811). Il y affirme que le médecin devait être un homme marié, faute de perdre la moitié de sa clientèle ${ }^{35}$. L'idée était répandue ${ }^{36}$ et l'impératif constitue un argument avancé dans ses lettres pour inciter une correspondante à l'accepter comme époux ${ }^{37}$. Ses propres démarches sur le marché matrimonial ne sont pas étrangères à sa stratégie professionnelle. Après un premier flirt avec une jeune écossaise, en 1772 il envisage simultanément d'épouser une amie d'enfance genevoise, et une sœur d'un ami d'étude de York, Jenny Withers. Les lettres d'amour qu'il écrit sont empreintes de références littéraires. Elles ont pour modèles Sophie et Julie, héroïnes de l'Emile et de la Nouvelle Héloïse de Rousseau ${ }^{38}$. Les motivations du jeune médecin apparaissent voilées d'une rhétorique de séduction classique. Certaines sont pragmatiques. La jeune Jenny Withers se présente comme une voie d'accès à

32. BGE, Ms fr 4152, Louis Odier à Mme Odier de Villas, Leyden, le 18.03.1773.

33. BGE, Ms fr 4155, Louis Odier à Andrienne Odier Lecointe, Genève, le 02.07.1787.

34. Voir à ce propos: Ph. Rieder, "The Physician Louis Odier and the Medical Market in Geneva (1774-1817)».

35. L. Odier, Les honoraires médicaux, p. 107.

36. Voir Ph. Rieder, M. Louis-Courvoisier, «Enlightened physicians», p. 594 sq.

37. BGE, Ms fr 4152, Louis Odier à Suzanne Baux, Londres, le 30.08. 1772.

38. Ph. Rieder, «Séduire en raisonnant». 
la pratique médicale en Grande-Bretagne. La fiancée potentielle était partie prenante du projet et estimait pouvoir l'aider à s'établir dans une ville du nord de l'Angleterre. Jenny ne trouve qu'un défaut à Louis "c'est de n'avoir pas autant de politesse extérieure et autant d'usage du monde qu'il en faut à un médecin", mais, ajouta-t-elle: "je me flatte qu'il me serait aisé de le polir " ${ }^{39}$. Le médecin épousera finalement la jeune Genevoise Suzanne Baux, pour des raisons sentimentales, comme il l'explique à sa mère ${ }^{40}$.

Le couple nouvellement marié s'établit à Genève en automne 1773. La jeune épouse n'apporte qu'une dot médiocre à son mari. Les revenus de la pratique sont faibles. De toute évidence, le médecin urbain devait faire ses preuves avant de gagner une clientèle. La pratique médicale des premières années d'Odier rapporte moins que celle de médecins de campagne dont les revenus sont connus: forts d'une clientèle captive, ceux-ci gagnaient un revenu décent dès la première année, soit plus de 1'000 livres tournois (l.t.) ${ }^{41}$. Odier n'atteindra une telle somme qu'après trois ans de pratique. La faiblesse des revenus du couple est compensée par le soutien des familles des époux. L'héritage recueilli par Odier à la mort de son père, en 1776, apporte une certaine autonomie au couple, qui dispose dès lors de quelque 65’000 l.t. C'est aussi l'époque où sa pratique progresse régulièrement, bien au-delà cette fois de ce que pouvait gagner un médecin de campagne. Après une décennie, Odier gagne plus de 5'000 1.t. par année. C'est une somme conséquente si on la compare à ce que gagnait alors un bon ouvrier (moins de 800 l.t.) ou même un ministre du culte (quelques 3’300 1.t.) ${ }^{42}$. L'activité déployée pour générer un tel revenu est importante. Le médecin travaillait sept jours par semaine sans ménager sa peine. En 1787, il explique à un ami: "Je vois près de 80 malades par semaine l'un dans l'autre, et cependant je ne gagne point encore assez pour subvenir à mes dépenses qui [se]

39. BGE, Ms fr 4152, Louis Odier à Mme Odier de Villas, Leyden, le 18.03.1773.

40. Ibid.

4I. C'est le cas d'Hilaire Raison à Fontenay-le-Comte (Vendée) et de Guillaume Martin à Romorantin (Sologne). R. Rouault de la vigne, "Une correspondance d'étudiants à la fin du XVIII siècle», p. 52; P. Treuttel, "Un médecin de Fontenay-le-Comte au début du XVIII ${ }^{\mathrm{e}}$ siècle».

42. Trois livres courants valent approximativement cinq livres tournois. H. Heyer, L'Eglise de Genève: 1555-1909, p. 80-82; B. Lescaze, Genève, sa vie et ses monnaies aux siècles passés, p. 86. 


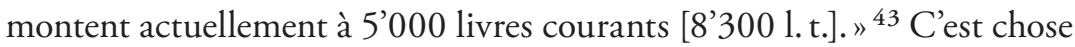
faite deux ans plus tard, lorsque son revenu avoisine les 9'000 1.t. Cette belle somme, signe de succès professionnel, dépasse de loin les 5’000 l.t. nécessaires, selon Isabelle Coquillard, aux médecins parisiens pour atteindre un équilibre budgétaire ${ }^{44}$. C'est aussi bien plus que ne gagnait un autre médecin de l'époque, Esprit Calvet, établi à Avignon, qui ne parvient à gagner 5'000 l.t. qu'après vingt ans de pratique ${ }^{45}$.

La fortune de Louis Odier est devenue conséquente après le décès de son père. Placée à $4 \%$, elle lui aurait rapporté plus de 2'500 l.t., soit plus de la moitié du revenu vital pour un médecin parisien. Le Genevois bénéficia également de revenus médicaux non négligeables, du moins jusqu’au début des années 1790. Ceci dit, il devait également faire face à des dépenses requises par son rang et sa situation familiale: un appartement dans le quartier le plus cher de la ville, cinq enfants à nourrir sans compter les cours particuliers, deux domestiques et un train de vie qui soit en rapport avec celui des familles les plus aisées de la ville. Le médecin estimait lui-même ses dépenses à près de 8’000 l.t. au début des années 1790 . Ses revenus, qui variaient en fonction de son avancement dans la carrière et de sa réputation, doivent donc être tempérés par le coût du maintien du train de vie auquel il devait répondre. De toute évidence, en dépit de son succès, Odier ne s'enrichit pas! Les dernières années du siècle sont même difficiles: la crise financière en France le ruine et une grande partie de sa clientèle quitte la ville suite aux troubles politiques. Le salut ne vient pas de la médecine, mais de son épouse, Andrienne Odier, qui se charge d'une pension assurant la subsistance de la famille dans les années difficiles ${ }^{46}$. Si les Odier retrouvent un niveau de vie confortable dès les premières années du $19^{\mathrm{e}}$ siècle, la reprise des affaires du médecin n'en est pas la seule raison: c'est en effet surtout grâce à une nouvelle entreprise d'Andrienne, qui importe et vend du tissu au détail ${ }^{47}$. Au final, l'étude de la vie économique du médecin confirme qu'il était possible de gagner de l'argent en pratiquant la médecine. Elle

43. BGE, Ms.fr. 4158, Louis Odier à Daniel De la Roche, le 04.04.1787. Voir aussi les chiffres qu'il insère la même année dans sa rubrique médicale dans le Journal de Genève.

44. I. Coquillard, «Les docteurs régents de la Faculté de médecine de Paris», p. 893.

45. L. Brockliss, Calvet's Web, p. 29.

46. Voir le récit de sa fille: BGE, Ms fr 5656, Souvenirs sur la vie privée de Louis Odier (t. 3), $12^{\mathrm{e}}$ cahier, p. 47-48.

47. BGE, Ms fr 5656, Souvenirs sur la vie privée de Louis Odier (t. 3), 10e cahier. 
signale cependant à quel point le marché médical était compétitif, les revenus médicaux variables et l'indépendance économique peu assurée. En outre, pour la fin de l'Ancien Régime encore, il s'agit d'envisager la vie économique d'un praticien dans un contexte plus large, celui d'une lignée, d'un couple, voire d'une famille.

\section{L'espace, les malades et le savoir médical}

Les revenus du médecin dépendent de son environnement social, ou, pour le dire autrement, s'il veut faire progresser ses revenus, il lui faut s'établir à proximité de familles aisées. Or, Odier était issu des quartiers marchands qui jouxtaient le port, au pied de la haute ville où se trouvait la clientèle souhaitée. Sa première installation prend place dans ce quartier, au bas de la rue de la Pélisserie, dans la maison de ses beaux-parents (1). En 1779, il quitte cet emplacement pour louer un appartement de la maison Jalabert, dans la haute ville: il s'agit de la deuxième maison en prenant à droite dans la Grand Rue depuis le haut de la Pélisserie (2). Il se trouve directement au-dessus de la maison de commerce de son frère aîné et du domicile de son frère cadet Jaques ${ }^{48}$. Il est socialement bien placé. Le médecin voisine désormais avec les grandes familles genevoises et c'est à dessein qu'il rechigne à quitter cet emplacement, comme il l'explique en 1779 à sa fiancée qui désirait s'établir au Bourg-de-four:

La solidité de mon établissement exige absolument que je sois logé à peu près au centre de la ville, moins pour moi qui ai bonnes jambes et à qui il est assez indifférent de marcher un peu plus ou un peu moins, que pour l'empressement des malades qui me consultent ${ }^{49}$.

La centralité est, si l'on observe la carte (ci-contre), davantage celle de la haute ville que celle de la ville elle-même. Prendre pied dans la haute ville représentait un objectif pour les jeunes médecins issus du monde du commerce, et plusieurs confrères d'Odier font comme lui dans les

48. No 408 au Teraillet. Archives d'Etat de Genève (désormais AEG), Recensement A 10 (1760-1782). Rôle de la dizaine de Monsieur l'ancien sindic Frédéric Guillaume Bonet (1775).

49. BGE, Ms fr 5654, Louis Odier à Andrienne Lecointe, [Genève], [s.d.]. 
décennies suivantes ${ }^{50}$. Aussi n'est-on guère surpris de trouver dans les années 1780 de bons clients habitant à proximité immédiate de la maison Jalabert. Pour donner deux exemples parmi d'autres, dans la maison voisine de la Grand Rue vit Jean-Paul Martin, un jeune homme de vingt-deux ans. En 1783, il règle une facture pour plus de 35 l.t. et, cinq ans plus tard, après une maladie qui requiert 90 visites du médecin, il verse au praticien la somme de 224 l.t. Ses parents, Pierre Martin et Marie Long, font leur entrée plus tard dans la comptabilité d'Odier, vraisemblablement sur recommandation de leur fils. Un autre voisin d'Odier, établi deux portes plus loin, en haut de la Pélisserie ${ }^{51}$, le colonel Charles Lullin (1715-1785), se révèle tout aussi bon client. Le vieux militaire recourt à Odier pour sa dernière maladie en 1785 . Le médecin encaisse plus de 312 l.t. pour ses services.

En été 1795 , lorsqu'il emménage plus près encore du centre géographique de la haute ville, dans le bâtiment de l'ancienne caserne, Odier trouve d'autres bons clients parmi ses voisins. Par exemple Pierre André Rigaud, conseiller et syndic, établi 244 rue des Granges, à quelques dizaines de mètres de l'appartement d'Odier, ou encore le régent Sébastien Esu, établi à la Cour Saint-Pierre, client régulier et père d'un enfant inoculé par Odier en 1800. La liste pourrait être poursuivie, mais elle ne sera jamais exhaustive. Quoi qu'il en soit, il est évident que le médecin côtoie les familles aisées en société, dans des sociétés savantes et dans la vie quotidienne. Le cas de $\mathrm{M}^{11 \mathrm{le}}$ Benelle en fournit une illustration. A la fin des années 1770, Odier alors veuf de fraîche date, envisage d'épouser cette voisine en secondes noces. Confronté au risque que sa descendance souffre d'une maladie héréditaire ${ }^{52}$, il renonce toutefois à l'alliance mais «il se lia depuis d'estime et d'amitié, avec M ${ }^{1 l e}$ Bennelle, après leur mariage à tous deux. Il fut toujours son médecin", rapporte sa fille. Et d'ajouter: «maman et elle, devenues voisines, devinrent amies, leurs filles le devinrent aussi $» 53$.

50. En 1783, Manget, Vignier et Vieusseux se trouvent encore établis dans la basse ville. Vieusseux et Vignier le rejoignent plus tard dans la haute ville. Voir AEG, Offices A, no 4, Role du Mag. Conseil des Deux-Cents, 1783.

51. AEG, Recensement A 10 (1760-1782). La troisième porte en descendant à droite $\left(\mathrm{n}^{\circ} 132\right)$ selon le dénombrement de 1782 .

52. Ph. Rieder and M. Louis-Courvoisier, «Introduction», p. 39 sq.

53. BGE, Ms fr 5656, Souvenirs sur la vie privée de Louis Odier (t. 3), p. 17 sq. 


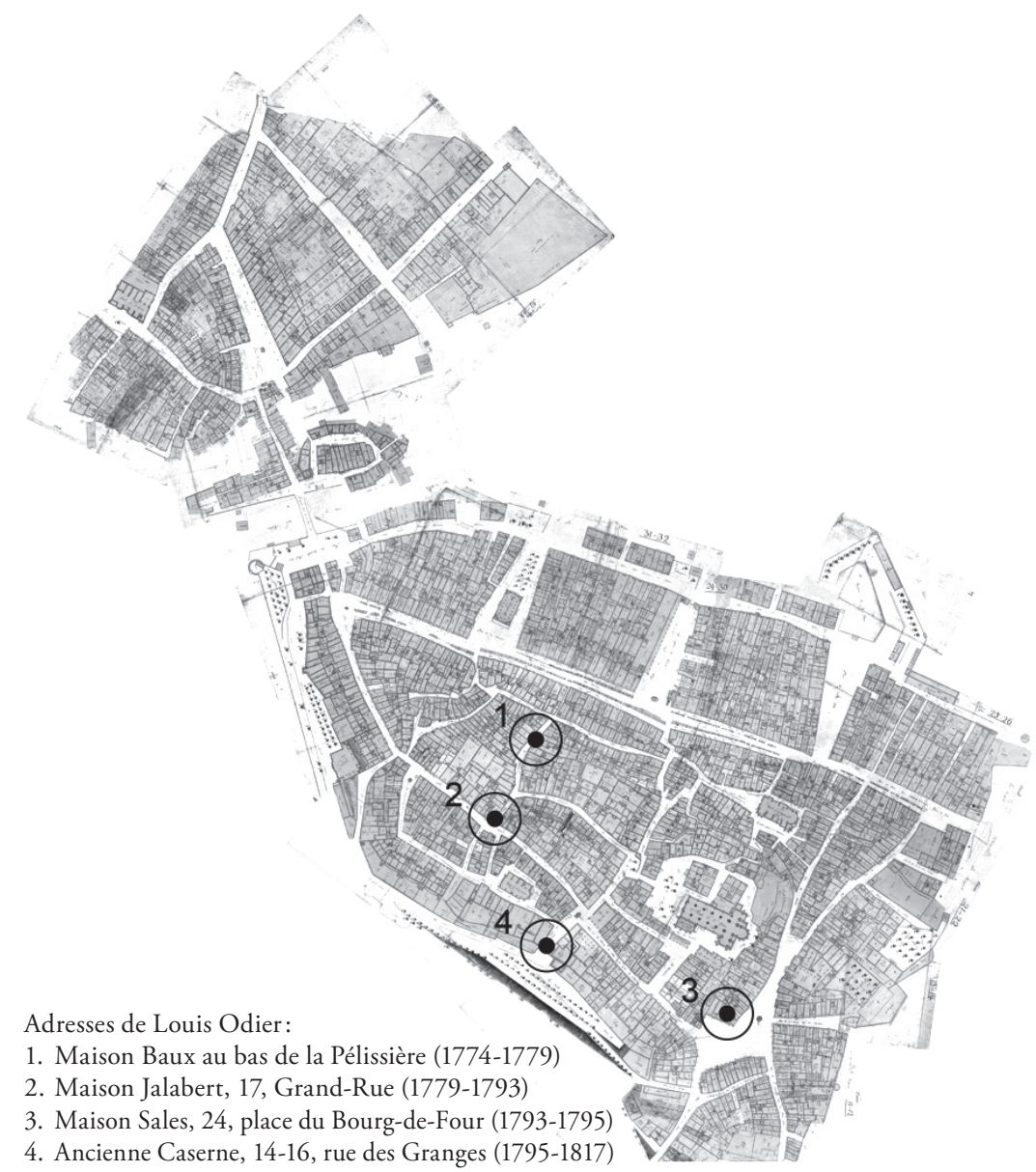

Plan de Genève réalisé par Jean-Michel Billon (1726)

Les réseaux sociaux et les réseaux de malades se superposent, le médecin se mouvant dans les mêmes milieux que ses patients. Son enracinement social a une influence non seulement sur le choix de sa clientèle, sur son porte-monnaie, mais aussi sur son activité scientifique. A la fin de l'Ancien Régime, l'essentiel de l'activité scientifique du praticien établi en ville consistait à observer ses patients, à transcrire le récit de leur cas et, ensuite, à en tirer des leçons pour améliorer sa pratique ou encore, pour contribuer au savoir médical. Or, dans la logique de la topographie médicale de son époque, les malades et les maladies de 
Genève étaient spécifiques au lieu ${ }^{54}$. De même, en centrant son activité sur quelques dizaines de familles bien établies, le médecin réduit la portée de ses observations à un groupe social particulier constitué de gens aisés. Oisifs pour certains d'entre eux, ils étaient tous habitués à un mode de vie raffiné auquel on associait des maladies bien distinctes de celles dont souffraient d'autres groupes sociaux. Les observations d'Odier de même que la façon de travailler qu'il conserva tout au long de sa carrière sont calquées sur le modèle de prise de cas qu'il avait appris à Edimbourg ${ }^{55}$. Plaçant l'accent sur les causes immédiates de la maladie, l'écriture du cas était séparée en trois sections. L'état de santé du malade à sa première rencontre avec le médecin forme le volet initial, où est rapporté le récit de l'histoire de la maladie elle-même. Un relevé chronologique des symptômes subséquents et des remèdes tentés forme la deuxième section. La dernière, intitulée "remarques", fournit l'occasion au médecin d'énoncer un avis sur le cas et de conclure sur ce qu'il pouvait en apprendre ${ }^{56}$. A l'exception de ce volet conclusif, le cas transcrit est donc vierge d'interprétation et peut être comparé à d'autres. C'est là une particularité qui distingue Odier - et ses collègues formés en Ecosse -, d'autres médecins contemporains qui avaient effectué leurs études en France. A l'image de Pierre Butini ou de Jean Verdier, par exemple, ces derniers intègrent les faits observés dans un récit qui n'exclut pas des renvois à d'autres auteurs, voire des citations, interprétant autant qu'ils décrivent leurs observations pour mener leur lecteur vers une interprétation définitive du cas ${ }^{57}$.

Au cours de sa carrière, Odier accumule près de 90 volumes de cas cliniques. Le médecin mobilise ses observations autant dans ses échanges avec ses collègues - dont il lit des extraits à la Société médicale, prête des volumes à ses collègues, et auxquels il fait référence à la fois dans ses lettres et dans ses publications ${ }^{58}$ - que dans ses travaux de médecine ${ }^{59}$.

54. G. Miller, "Airs, Waters, and Places” in History»; J.-P. Peter, "Aux sources de la médicalisation, le regard et le mot».

55. Voir G. B. Risse, Hospital life in Enlightenment Scotland.

56. Ph. Rieder, "Writing to fellow physicians».

57. Pour leurs recueils de cas, voir respectivement: Musée d'histoire des sciences (Genève), Z 97; Wellcome Library, Ms 4920 et Ms 4921.

58. MHS, SM Ms. 8, Procès-verbaux de la Société médico-chirurgicale (1806-1811), p. 74, le 19 août 1809; Ms fr 4158, Louis Odier à Daniel De la Roche, [1793].

59. L. Odier, "A Clinical History of Acute Rheumatism», p. 49-52. 
Mais qu'y apprend-on? L'historien pressé y cherche les prémices de la démarche anatomo-pathologique qui s'épanouit quelques décennies plus $\operatorname{tard}^{60}$. Une lecture attentive des publications d'Odier révèle un intérêt pragmatique pour les informations sur les thérapies les plus efficaces, incluant toute indication susceptible de lui être utile dans sa pratique. Les quatre cas qu'il publie en 1779 dans l'Histoire de la Société royale de médecine en fournissent un bon exemple. Ils sont censés permettre la reconstruction de l'histoire clinique de l'hydrocéphale (ou malade souffrant d'un épanchement de liquide dans le crâne). Chaque cas comprend une brève anamnèse et une histoire succincte des symptômes constatés lors de la première visite du praticien. Suit le récit de la maladie au quotidien avec, pour chaque jour, les prescriptions données et les thérapies tentées. Le cas se termine par une section «remarque» dans lequel le médecin cherche à comprendre l'évolution de la pathologie et l'efficacité des différents remèdes ${ }^{61}$. L'organisation des cas reprend donc bien le format de transcription d'observations apprise à Edimbourg. Le fait que le médecin privilégie les quatre cas qui virent le malade se remettre trahit l'accent particulier mis sur la thérapeutique. Le récit clinique de la maladie qu'il propose s'articule en fin de compte autour de quatre cas qui n'ont pas été autopsiés - le médecin ne peut prouver qu'ils souffraient de la maladie - et sert de fait à démontrer la capacité du médecin à guérir des malades! C'est là une orientation commune parmi les praticiens, mise en avant par Baglivi déjà, et lisible dans les échanges professionnels ${ }^{62}$. L'exemple incite à intégrer plus largement l'étude des écrits médicaux dans des stratégies de carrière, voire de publicité médicale: la production médicale n'a pas pour seule finalité la progression des savoirs, elle répond aux ambitions et aux intérêts de ses auteurs, de même qu'aux attentes supposées du lectorat visé. "Tant que les gens du monde ne seront pas appelés à lire vos ouvrages", confie Odier à un collègue, "ils feront peu de sensation, ou s'ils en font parmi les médecins, cela ne vous sera pas extrêmement avantageux $"{ }^{63}$. Publier des articles médicaux constitue un moyen de faire progresser sa pratique. Ainsi, les cas rapportés dans l'article scientifique d'Odier sont pensés comme

6o. M. Foucault, Naissance de la clinique.

6I. L. Odier, «Mémoire sur l'hydrocéphale interne», p. 213-232.

62. Voir J. Pigeaud, «La Renaissance hippocratique au XVIII ${ }^{\mathrm{e}}$ siècle», p. 584 sq.

63. BGE, Ms.fr. 4158, Louis Odier à Daniel De la Roche, le 1.12.1794. 
des faire-valoir du médecin ou, pour reprendre l'heureuse formule de Jean-Pierre Peter, servent à «mettre en spectacle la vertu, l'art et les pouvoirs du médecin. ${ }^{64}$

\section{L'échelle individuelle: débouchés historiques}

L'étude historique d'un fond d'archives issu d'une "vie biographique» représente un point de départ qui ancre la démarche historienne dans une réalité vécue à partir de laquelle différentes voies sont possibles. Dans cet article, les thèmes de la vocation, de la vie économique et du rapport au savoir d'un médecin ont été choisis - parmi d'autres- à titre d'exemple. Les différents éclats de vie qui éclairent le propos y prennent sens non seulement à partir des contextes thématiques et sociaux-culturels établis par ailleurs, mais en fonction d'autres données biographiques, c'est-à-dire d'éléments antérieurs pris dans le parcours de vie du sujet. Le choix de mettre en avant le potentiel de ces écrits autour de thèmes qui dépassent l'histoire de l'individu permet de sortir de la compartimentation propre à une biographie littéraire articulée autour des différentes étapes de la vie du sujet et organisée autour du sens général qu'il s'agirait d'en tirer. L'intelligibilité historique prend ici le dessus sur la nécessité d'imprimer une cohérence narrative. Le constat est banal au vu des travaux de l'école microhistorique, mais stimulant dans le champ de l'histoire de la médecine où prévaut une forte méfiance à l'égard de la biographie.

Au-delà du point de vue subjectif de l'acteur choisi, la perspective individuelle permet de valoriser la cohérence sociale et culturelle qui lie différents thèmes ou plus précisément, les connexions qui permettent d'en rendre compte. Elle permet d'appréhender des stratégies d'acteurs, des logiques sociales et des réalités culturelles autrement inaccessibles. Ancrée dans un contexte alimenté par des sources riches et elles-mêmes bien contextualisée, elle invite, aussi paradoxal que cela puisse paraitre au premier abord, à prendre de la distance par rapport aux affirmations du sujet biographique lui-même. Songeons à la déclaration de Louis Odier selon laquelle son père aurait choisi une carrière pour lui, destinée à donner de lui-même l'image d'un fils docile. Ou à ses doléances

64. J.-P. Peter, «L'histoire par les oreilles», p. 298. 
économiques. Lorsqu'il se plaignait d'être pauvre, le praticien entendait plutôt que sa situation économique le contraignait à travailler pour vivre selon son rang. Avec pour conséquence la plus évidente l'obligation de privilégier les malades aisés, ceux qu'il connaissait, sa production scientifique semblant dès lors, en schématisant quelque peu, autant motivée par la nécessité de gagner cette clientèle que par l'espoir de faire progresser le savoir médical.

Au nombre des apports de l'approche biographique, il faut compter les nouvelles problématiques qu'elle ouvre. Celle de la vocation médicale dont il a été question pour commencer suggère l'importance de la littérature - et surtout d'une poignée d'auteurs - dans le façonnement de points de vue qui conditionnent les décisions prises: le cas d'Odier signale combien les jeunes lettrés pouvaient se montrer perméables à la production des Lumières. Cette question amène en outre à repenser un ensemble de stratégies sociales dans un contexte culturel donné. Si le résultat est peu étonnant - établir une corrélation entre l'anticléricalisme croissant et la baisse des vocations ecclésiastique relève du bon sens l'intérêt tient dans la démonstration. Comment donner de la substance à une telle corrélation si ce n'est à partir d'une échelle individuelle? En suivant un raisonnement analogue, l'histoire de l'économie de la pratique d'Odier constitue un bon exemple des difficultés économiques que pouvait connaître un médecin. Ses tribulations financières révèlent l'importance du rôle de son épouse, Andrienne Odier. Le médecin d'Ancien Régime était du reste traditionnellement un homme marié, le célibat pouvant générer un développement moins rapide d'une pratique ${ }^{65}$. Autour d'Odier apparaissent les différents moyens que pouvait mobiliser une famille médicale pour compléter ses revenus. Enfin, une analyse de la production médicale du praticien souligne l'importance de la formation dans la carrière médicale, et l'adéquation de la production scientifique avec la clientèle du médecin et donc, par la force des choses, avec sa géolocalisation: d'un point de vue médical, le praticien s'impose comme un spécialiste des maladies de ses patients, et ses publications scientifiques tendent à le mettre en valeur en tant que tel.

D'un point de vue historiographique, le survol réalisé dans cette contribution tend à dévoiler un niveau de réalité difficile à appréhender par le biais d'autres sources, tout en suggérant des pistes de recherches.

65. Voir par exemple L. Brockliss, Calvet's Webb. 
Il ne s'agit pas d'offrir l'histoire définitive d'un parcours individuel. L’approche prônée tend à alimenter des problématiques plus larges sur la pratique médicale et la figure du médecin. La biographie sert ici de révélateur et incite à opérer des allers-retours avec d'autres chantiers (l'histoire économique, l'histoire familiale, etc.) et d'autres contextes particuliers (le cadre des études, de la pratique, etc.). La poursuite du travail peut mener plus loin. L'étude des motivations des étudiants toujours plus nombreux en médecine à la fin de l'Ancien Régime pourrait permettre de clarifier le statut culturel de la médecine à cette époque. Une extension de la problématique autour de l'économie de la pratique à d'autres individus et à d'autres milieux inviterait à reconsidérer le statut économique des médecins avant les assurances sociales en prêtant une attention particulière à l'importance du statut économique du médecin et de sa famille. La nature détaillée de la pratique du médecin permet de développer des problématiques sur le savoir médical et de repenser ses relations avec ses malades, voire d'envisager une histoire de la relation thérapeutique.

Un tel potentiel, encore théorique à ce stade, ne doit pas occulter les difficultés inhérentes à la démarche. L'approche biographique inscrit inévitablement, semble-t-il, la publication de ses résultats dans un genre, celui de la biographie, et comporte de ce fait le risque - dont témoigne le cas de Jacalyn Duffin mentionné plus haut -, d'être mal lu ou, surtout, peu lu par des historiens professionnels. La multiplication de problématiques différentes dans une même publication (ou livre) peut décourager de la lecture des spécialistes des domaines abordés. Comment présenter le travail à d'autres que les seuls lecteurs de biographies? Mais aussi, comment raconter l'histoire d'un individu sans proposer un début et une fin, sans offrir une lecture "définitive" de la vie d'un sujet? Répondre à ce double questionnement constitue un défi en soi, un défi qui paraît toutefois à la hauteur de l'intérêt historiographique et des apports possibles de l'approche biographique à l'histoire de la médecine.

Philip Rieder

Université de Genève 


\section{BIBLIOGRAPHIE}

Balster, Wolfgang, Medizinische Wissenschaft und ärztliche Praxis im Leben des Bochumer Arztes Karl Arnold Kortum (1745-1824), Bochum, s. n., 1990.

Bourdieu, Pierre, "L'illusion biographique», Actes de la recherche en sciences sociales, 62 (1986), p. 69-72.

Brockliss, Laurence, "The Medico-religious Universe of an Early Eighteenth-Century Parisian Doctor: the Case of Philippe Hecquet", in The Medical Revolution of the Seventeenth Century, ed. by Roger French, Andrew Wear, Cambridge, Cambridge University Press, 1994.

-, Calvet's Web: Enlightenment and the Republic of letters in Eighteenthcentury France, Oxford, Oxford University Press, 2002.

Broman, Thomas H., "Rethinking Professionalization: Theory, Practice, and Professional Ideology in Eighteenth-Century German Medicine", The Journal of Modern History, 67 (1995), p. 835-872.

Coquillard, Isabelle, «Les docteurs régents de la Faculté de médecine de Paris et la fourniture de soins aux "bons pauvres malades" dans les paroisses parisiennes (1644-1791)», Revue historique, 668 (2013), p. 875-904.

De Renzi, Silvia, "Medical Competence, Anatomy and the Polity in Seventeenth-century Rome", Renaissance Studies, 21 (2007), p. 551-567.

Demaitre, Luke, "Theory and Practice in Medical Education at the University of Montpellier in the Thirteenth and Fourteenth Centuries", Journal of the History of Medicine and Allied Sciences, XXX (1975/2), p. 103-123.

Duffin, Jacalyn, "La mauvaise herbe”: Unwanted Biographies Both Great and Small», in The History and Poetic of Scientific Biography, ed. by Thomas Söderqvist, Oxford, Oxford University Press, 2005, p. 185-197.

Foucault, Michel, Naissance de la clinique, Paris, PUF, 1972. 
Gautier, Léon, La médecine à Genève jusqu'à la fin du XVIII' siècle, Genève, Julien, 1906.

Goubert, Jean-Pierre, Médecins d'hier, médecins d'aujourd'hui. Le cas du Docteur Lavergne (1756-1831), Paris, Publisud, 1992.

Gregory, John, Lectures on the Duties and Qualifications of a Physician, Edinburgh, Strahan \& Cadell, 1772.

Guillemain, Hervé, "Devenir médecin au XIXe siècle: vocation et sacerdoce au sein d'une profession", Annales de Bretagne et des Pays de l'Ouest, 116 (2009), p. 109-123.

Heyer, Henri, L'Eglise de Genève: 1555-1909, Genève, Slatkine, 1974 (1909).

Jubert, Gérard, "L'homme Renaudot", in Théophraste Renaudot l'homme, le médecin, le journaliste, 1586-1986, éd. par Jean Imbert, Paris, Institut français de presse et des sciences de l'information, 1987, p. 9-23.

Lescaze, Bernard, Genève, sa vie et ses monnaies aux siècles passés, Genève, Crédit Suisse, 1981.

Loux, Françoise, Pierre-Martin de la Martinière un médecin au XVIII siècle, Paris, Imago, 1988.

Mandressi, Rafael, «Le passe, l'enseignement, la science: Felix Vicq d'Azyr et l'histoire de la medecine au XVIII' siecle», Medicina nei secoli, 20 (2008), p. 183-212.

Nye, Mary Jo, "Scientific Biography: History of Science by Another Means?», Isis, 97 (2006), p. 322-329.

Miller, Geneviève, "“Airs, Waters, and Places" in History», Journal of the History of Medicine and Allied Sciences, XVII (1962), p. 129140.

Odier, Louis, «Mémoire sur l'hydrocéphale interne, ou hydropisie des ventricules du cerveau", in Histoire de la Société royale de médecine, Paris, P.-D. Pierres, 1779, p. 194-232.

—, "A Clinical History of Acute Rheumatism. Histoire clinique du Rhumatisme aigu par John Haygarth", Bibliothèque Britannique, 34 (1807), p. 47-167.

—, Les honoraires médicaux et autres mémoires d'éthique médicale, éd. par Philip Rieder, Micheline Louis-Courvoisier, Paris, Classiques Garnier, 2011.

Peter, Jean-Pierre, "L'histoire par les oreilles", Le temps de la réflexion, 1 (1980), p. 273-314. 
—, "Aux sources de la médicalisation, le regard et le mot: le travail des topographies médicales", in Populations et cultures, études réunies en l'honneur de François Lebrun, Rennes, Amis de François Lebrun, 1989, p. 103-111.

Pigeaud, Jackie, "La Renaissance hippocratique au XVIIIe siècle», in Hippokratische Medizin und antike Philosophie, hrsg. von Pierre Wittern, Renate Pellegrin, Zurich, Olms Weidmann, 1996, p. 583-610.

Pilloud, Séverine, Les mots du corps: l'expérience de la maladie dans les lettres de patients à un médecin du XVIII e siècle: Samuel Auguste Tissot, Lausanne, Editions BHMS, 2013.

Le Roy Ladurie, Emmanuel et al., Le siècle des Platter, 3 vol., Paris, Fayard, 1995-2006.

Prevost, Pierre, Notice de la vie et des écrits de Louis Odier, Docteur et Professeur de médecine, Correspondant de l'Institut de France, Membre de plusieurs Sociétés savantes, Paris, Paschoud, 1818.

Rieder, Philip, "Séduire en raisonnant: les conquêtes épistolaires de Louis Odier (1748-1817), médecin et citoyen de Genève», in La correspondance familiale en Suisse romande aux XVIII et XIX ${ }^{e}$ siècles, éd. par Philippe Henry, Jean-Pierre Jelmini, Neuchâtel, Alphil, 2006, p. 75-95.

-, "The Physician Louis Odier and the Medical Market in Geneva (1774-1817)», Gesnerus, 69 (2012), p. 54-75.

—, «Writing to fellow physicians: literary genres and medical questions in Louis Odier's (1748-1817) correspondence", in Medicine and Narration in the Eighteenth Century, ed. by Sophie Vasset, Oxford, Voltaire Foundation, 2013, p. 47-63.

Rieder, Philip, Louis-Courvoisier, Micheline, "Enlightened Physicians: Setting out on an Elite Academic Career in the Second Half of the Eighteenth Century", Bulletin for the History of Medicine, 84 (2010), p. 578-606.

—, "Introduction: genèse d'une éthique médicale», in Les honoraires médicaux et autres mémoires d'éthique médicale, éd. par Philip Rieder, Micheline Louis-Courvoisier, Paris, Classiques Garnier, 2011, p. 13-42.

Risse, Guenter B., Hospital life in Enlightenment Scotland: Care and teaching at the Royal Infirmary of Edinburgh, Cambridge, Cambridge University Press, 1986. 
Roche, Daniel, "Talents, raison et sacrifice: l'image du médecin des Lumières d'après les Eloges de la Société royale de médecine (1776-1789)», Annales ESC, 32 (1977), p. 866-886.

Rouault DE LA Vigne, René, "Une correspondance d'étudiants à la fin du XVIII siècle. Les études et l'installation d'un médecin", Revue des sociétés savantes de Haute-Normandie, 31 (1963), p. 33-62.

Siraisi, Nancy G., Medieval and Early Renaissance Medicine: an Introduction to Knowledge and Practice, Chicago, University of Chicago Press, 1990.

-, The clock and the mirror: Girolamo Cardano and Renaissance medicine, Princeton, Princeton University Press, 1997.

Söderquivist, Thomas, Science as autobiography: the troubled life of Niels Jerne, New Haven, Yale University Press, 2003.

Steinke, Hubert, «Der Junge Arzt une seine Patienten: Albrecht von Hallers Praxis in Bern 1731-1736", in Arztpraxen im Vergleich: 18.-20. Jahrhundert, hrsg. von Elisabeth Dietrich-Daum, Martin Dinges et al., Innsbruck, Studienverlag, 2008, p. 79-86.

Stolberg, Michael, Homo patiens, Köln, Böhlau, 2003.

Tissot, Samuel Auguste, L’onanisme, éd. par Christophe Calame, Paris, Editions de la Différence, 1991 (1760).

Treuttel, Pierre, "Un médecin de Fontenay-le-Comte au début du XVIII siècle", Annales de Bretagne et des pays de l'Ouest, 90 (1983), p. 19-33.

\section{Illustration}

Plan de Genève réalisé par Jean-Michel Billon (1726), Archives d'Etat de Genève (AEG), Cadastre A2. Scan réalisé par l'Inventaire des monuments d'art et d'histoire du canton de Genève (IMAHGE). 